\title{
CHARACTERIZATION OF HEAT CONDUCTIVITY OF ECCENTRICALLY ROTATING HEAT PIPES USED FOR COOLING OF MOTOR SPINDLES
}

\author{
B. Denkena ${ }^{1}$, B. Bergmann ${ }^{1}$, K. Kono², R. Ishiguro², H. Klemme ${ }^{\text {* }}$ \\ ${ }^{1}$ Leibniz Universität Hannover, Institute of Production Engineering and Machine Tools (IFW), Garbsen, Germany \\ ${ }^{2}$ Makino Milling Machine Co., Ltd., Kanagawa, Japan \\ ${ }^{\star}$ Corresponding author; e-mail: klemme@ifw.uni-hannover.de
}

\begin{abstract}
Heat losses within motor spindles lead to undesired effects such as machining inaccuracies and decreasing lifetime of the motor and bearings. To reduce thermal loads, complex shaft cooling concepts with costly sealing techniques exist. For this reason, a novel, less costly cooling concept has been developed based on heat pipes with high thermal conductivity and fin-shaped heat exchangers. The design and integration of these heat exchanger elements into a motor spindle is carried out using the finite element method. The aim is to optimize the efficiency of the heat pipes and heat exchangers for optimal shaft cooling performance. For a simulation-based development of a prototype spindle, unknown thermal characteristics of the heat transfer elements must be determined. In this paper, the determination of the thermal conductivity of the heat pipes is described. The determination of conductivity is done experimentally. First, the developed test rig and the applied procedure for the determination of the conductivity are shown. Subsequently, the experimental results are presented and discussed. Two types of heat pipes were analyzed: Copper heat pipes with sintered wick structure and nickel-plated copper heat pipes with axial grooves. The influences of rotational speed, heat flow rates and the angle between the heat pipe and main axis of rotation were investigated. The results indicate a distinct dependency of the conductivity on the varied parameters. However, changes of parameter values have very different quantitative and qualitative effects on the determined conductivities depending on the type of heat pipe.
\end{abstract}

\section{Keywords:}

Heat pipe; Eccentric rotation; Spindle cooling; Thermal conductivity

\section{INTRODUCTION}

When electrical energy is converted into mechanical energy, electrical losses occur in motor spindles which result in heat. Both bearing as well as fluid friction cause additional thermal loads, which affects the thermo-mechanical spindle behavior [Gebert 1997]. The achievable accuracy in machining is significantly affected by these thermal loads. The thermally induced expansion of the spindle leads to geometric errors on the workpiece. The heating of the shaftbearing system causes undesired variation in the bearing preload. In the worst case scenario, especially excessive heating of the bearing inner rings can lead to an excessive decrease in bearing clearance. As a result, a bearing can suddenly become damaged. Heating of the bearing inner rings or the rotating train also limits the achievable maximum speed or the previously adjustable bearing preload. Therefore, the achievable productivity of the spindle is limited. The cooling of the rotary train and especially of the bearing inner rings is therefore a significant challenge in the development of more powerful motor spindles [Denkena 2020a].
Various cooling concepts have been developed over the past decades to reduce thermally induced loads on the rotary train. An overview of these concepts can be seen in [Denkena 2020a]. The most effective method has proven to be the liquid-based cooling of the shaft by means of rotary joints. However, such concepts are often associated with very high purchase and operating costs. The leakage of rotary unions is the second most frequent cause of failure in motor spindles [Abele 2011]. For these reasons, a new approach to cooling spindle shafts based on highly conductive heat pipes (HP) is being investigated within the scope of a research project. The HP are arranged eccentrically around the main axis of rotation in the front and rear spindle area $\begin{array}{llll}\text { Rear } & \text { Rear } & \text { Stator jacket Front jacket } & \mathbf{z}\end{array}$ jacket heat pipe 7 cooling cooling
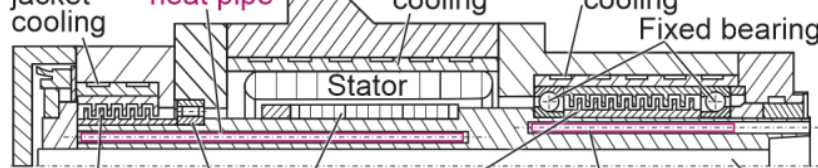
Rear heat Rear Rotor Front heat Front Shaft exchanger bearing exchanger heat pipe KI/102275@ IFW

Fig. 1 : Illustration of concept for shaft cooling based on heat pipes according to [Denkena 2018] 
to allow the integration of a tool clamping system in the hollow shaft. The HP conduct the heat to finned heat exchangers. The heat is dissipated convectively by non-rotating fins using the obligatory jacket cooling. A schematic illustration of this concept is shown in Fig. 1.

In order to optimize the cooling potential of this concept, a simulation model of the spindle was developed. This model is described in [Denkena 2020b]. In order to achieve a realistic simulation, various parameters need to be identified, such as heat losses of individual heat sources, cooling capacities of the heat sinks and heat transfer coefficients between spindle components. In addition, the thermal conductivities of the applied HP are unknown but highly relevant parameters which must be determined. Consequently, the aim of this study is to experimentally determine the thermal conductivities of eccentrically rotating HP, especially at high speeds. The procedure is shown in this paper. First, the operational principle of eccentrically rotating HP is discussed in Section 2. Furthermore, other state of the art concepts are presented, in which shaft cooling is achieved by using HP. In Section 3, the test rig is described as well as the procedure for the determination of the conductivities in this study. In Section 4, the evaluation of the experiments is shown. The experiments were carried out for two different HP types with diverse capillary structures under varying dynamic and thermal boundary conditions. By comparing two different types of heat pipes, the effect of the capillary structure on heat transfer during fast eccentric rotation shall be evaluated.

\section{HEAT PIPE SHAFT COOLING}

\subsection{Operation of heat pipes}

Heat pipes are closed, evacuated hollow structures containing a working fluid, typically deionized water [Li 2020b]. The wall of a HP is made of a thin, well heat conducting material. Such HP can be operated within a temperature range of $0^{\circ} \mathrm{C}$ and $340^{\circ} \mathrm{C}$ [Reay 2014]. Frequently, there is a capillary structure saturated with working fluid inside the tube. By using the evaporation and condensation enthalpy of the working fluid, a high heat flux density can be achieved. This density can be higher than that of geometrically identical copper bars by a factor of $10^{3}$ [Gebert 1997]. By varying the working fluid, the geometric parameters, the capillary structure, the capillary, and tube materials, heat pipes can be designed for a wide range of applications.

A HP can be divided into three zones (Fig. 2). By applying heat in the vaporization zone, the working fluid vaporizes into the adiabatic transport zone until it is first oversaturated and finally superheated. The vapor is driven into the condensation zone by a temperature or pressure gradient. The condensation enthalpy of the working fluid is released as heat energy. The vaporous fluid condenses and is returned into the evaporation zone. This recirculation is achieved by capillary, gravity or centrifugal forces. A HP only functions if there is a temperature gradient between the evaporation

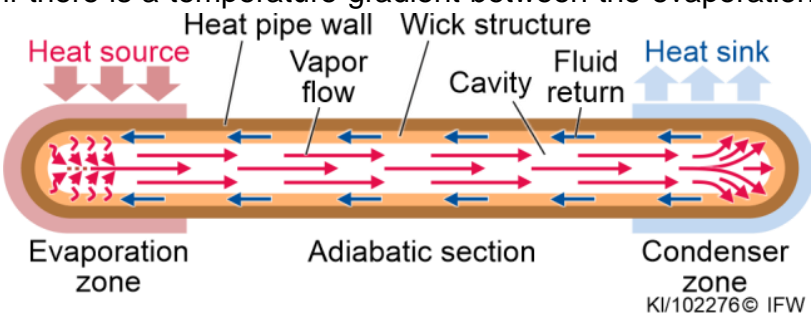

Fig. 2 : Design and operating principle of a heat pipe and condensation zones.
The transferable heat quantity is significantly influenced by the available fluid filling quantity, as the fluid film acts as a thermal resistance during evaporation [Faghri 1995]. Accordingly, many papers (e.g. [Hassan 2017; Song 2003; Semena 1991]) have found that a thinner film allows increased heat transfer. However, Li et al. [Li 1993] conclude that there is an optimal fluid filling quantity for each operating condition. This quantity depends largely on the respective operating parameters as well as thermal and geometrical boundary conditions, such as the dimensions of the pipe [Faghri 1995], the prevailing temperatures in the evaporator and condensation zones [Song 2008; Ling 2001], the lengths of these zones [Kimura 2005] and the properties of the capillary structure [Niekawa 1981], fluid and pipe material [Faghri 1995].

Heat pipes can be operated both when stationary and while rotating. When the HP rotates, the centrifugal force acts on the fluid. The fluid is transported axially by the associated fluid film flattening. Such a flattening favors the heat transfer [Jankowski 2007; Lian 2016; Song 2003]. As a result of the increasing centrifugal force acting on the fluid film, the amount of heat that can be transferred increases due to the greater axial force component. This is also observed in [Daniels 1975; Chatterjee 2018; Groll 1978; Li 1993].

The fluid return is supported by the centrifugal force using a conical internal structure with an angle a or by tilting the HP axis towards the main axis of rotation with a tilt angle $a_{c}$. During rotation, an additional centrifugal acceleration with an axial component $\omega^{2} \cdot r \cdot \sin \left(a_{c}\right)$ will then act on the fluid. In Fig. 3, the equilibrium forces on a control volume at constant rotational speed $n$ is illustrated according to [Faghri 1995]. The influence of a possible capillary structure is not considered. However, rotating HP often do not have capillary structures, as the centrifugal force-induced fluid return is dominant [Asias 2007]. However, based on the current knowledge, it is understood that the presence of a capillary structure can have a positive effect on heat transfer. This is due to the resulting increase in the surface area available for evaporation or condensation of the working fluid [Salinas 1991; Liang 2019a; Reay 2014].

The static equilibrium of forces in $\mathrm{x}$ and $\mathrm{y}$ direction is expressed by Eqs. 1 and 2, respectively:

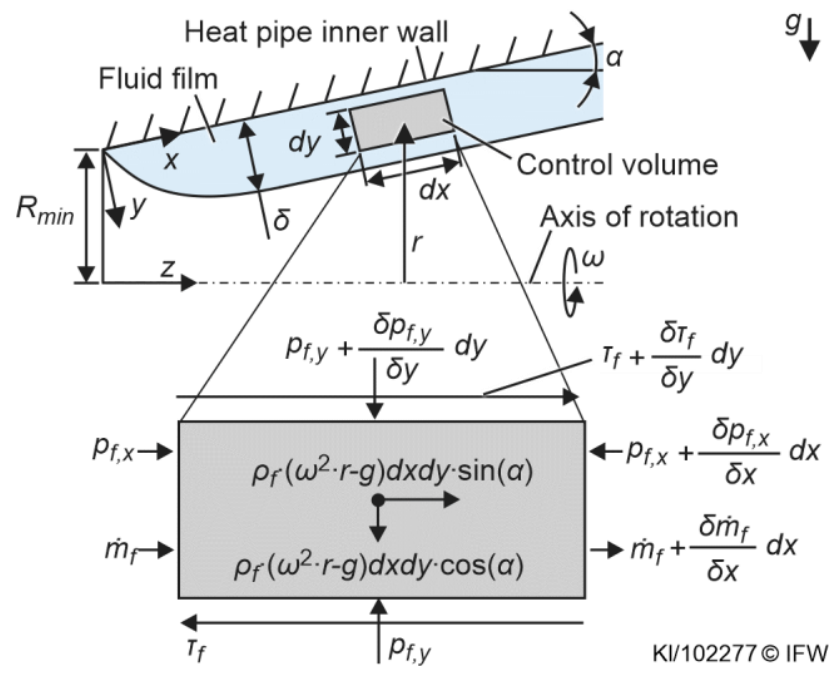

Fig. 3 : Equilibrium of forces on a fluid control volume at constant rotational speed

$\frac{\delta \tau_{f}}{\delta y}-\frac{\delta p_{f}}{\delta x}+\rho_{f} \cdot\left(\omega^{2} \cdot r-g\right) \cdot \sin (\alpha)=0$

$-\frac{\delta p_{f}}{\delta y}-\rho_{f} \cdot\left(\omega^{2} \cdot r-g\right) \cdot \cos (\alpha)=0$ 
The internal fluid shear stress is represented by $\tau_{f}$, the angular velocity by $\omega$, the taper angle of the cone by $\alpha$ and the density of the fluid by $\rho_{f}$. The local distance of the fluid to the rotation axis $r$ is determined by Eq. 3 based on the minimum distance between rotation axis and the inner HP wall $R_{\text {min. }}$

$$
r=R_{\min }+x \cdot \sin \left(\alpha_{c}\right)-y \cdot \cos \left(\alpha_{c}\right)
$$

When a HP is oriented horizontally, the fluid accumulates in the lower part, assuming Eq. $1 g>\omega^{2} \cdot r$ applies. This is unless the HP have a capillary structure which distributes the fluid around the circumference of the pipe at low centrifugal accelerations (Fig. 4). As a result of the fluid accumulation, a considerable section of the inner surface of the pipe remains dry. If $g<\omega^{2} \cdot r$ applies, the fluid is conveyed into the outer area of the HP towards the pipe wall during eccentric rotation with increasing centrifugal force. The accumulation of the fluid increases the fluid film thickness locally. This increases the thermal resistance, which results in a reduction of the evaporation rate.

\subsection{Shaft cooling concepts based on heat pipes}

Research that deals with eccentrically rotating HP origi-

\begin{tabular}{|c|c|c|}
\hline $\begin{array}{c}\text { Eccentric } \\
\text { rotation }\end{array}$ & 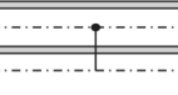 & $\begin{array}{l}\text { Axis of } \\
\text { rotation }\end{array}$ \\
\hline \multicolumn{3}{|c|}{ Fluid accumulation } \\
\hline$g>\omega^{2} \cdot r ; \omega=0$ & $g>\omega^{2} \cdot r ; \omega>0$ & $g<\omega^{2} \cdot r ; \omega>>0$ \\
\hline$\otimes z \multimap \square$ & & \\
\hline & & \\
\hline
\end{tabular}

\section{Fig. 4 : Distribution of the fluid in an eccentrically rotating} heat pipe at different peripheral speeds

nates mainly from the 1960 s to 1980 s. The reason for this was the identified potential for cooling induction motors with hollow shafts. However, due to the increasingly relevant topic of cooling motor spindles, such maintenance and energy-efficient concepts have again become the focus of research in recent years [Denkena 2020a]. In [Mochizuki 1980], eccentrically rotating HP with a length of up to $I_{H P}$ $=452 \mathrm{~mm}$ without capillary structure are investigated analytically and experimentally. The maximum analyzed rotational speed is $n=480 \mathrm{~min}^{-1}$ which corresponds to a centrifugal acceleration of $a_{c}=251 \cdot \mathrm{g} \mathrm{m} / \mathrm{s}^{2}$. It is found that the heat conductivity is facilitated by the increase of rotational speed. This effect is also shown in [Hassan 2017]. In [Niekawa 1981] eccentrically rotating HP with a length of $I_{H P}$ $=3,000 \mathrm{~mm}$ are analyzed. Here, heat pipes without internal structure and with axial groove structures are considered separately. Contrary to the previously mentioned work, a decrease in heat conductivity with increasing rotational speed $n$ between $20 \mathrm{~min}^{-1}$ and $180 \mathrm{~min}^{-1}\left(a_{c}=196 \cdot \mathrm{g} \mathrm{m} / \mathrm{s}^{2}\right)$ is determined for both types. The stated reason is the drying out of a section of the evaporator zone and the increased fluid film thickness due to the centrifugal force induced fluid accumulation, respectively.

In [Li 2018], a concept is presented for cooling of a grinding spindle with several eccentrically rotating ring-shaped HP in the spindle shaft. Cooling is achieved by feeding an air stream onto the rear shaft section. In this paper, the heat transfer performance is investigated experimentally. It is found that the thermal conductivity is dependent on the fluid filling quantity. With comparatively high fluid filling quantities, the thermal conductivity increases up to a heat flow rate of $225 \mathrm{~W}$. With small fluid filling quantities, however, a reduction of the conductivity occurs with noticeably smaller heat flow rates. The simulated maximum temperature of the spindle at a rotational speed of $5,000 \mathrm{~min}^{-1}$ is reduced from $78.4{ }^{\circ} \mathrm{C}$ to $56.2{ }^{\circ} \mathrm{C}(28 \%)$. In [Li 2020a], the cooling effect of this concept is evaluated using a test rig. The temperature of the evaporation section is decreased from $99^{\circ} \mathrm{C}$ to $57^{\circ} \mathrm{C}$ $(42 \%)$ for a thermal load of $150 \mathrm{~W}$ and a rotational speed of $1,000 \mathrm{~min}^{-1}$. In [Liang 2019b], [Liang 2019a] and [Liang 2020] a concept for cooling a grinding spindle with eccentrically rotating HP at an angle of $\alpha_{H P-S}=0^{\circ}$ is shown. Cooling is also achieved here by blowing air onto the rear shaft surface. In [Liang 2019b] and [Liang 2019a] the heat conductivity properties of the applied HP are investigated. In this work, an increase of the thermal conductivity with increasing heat flow rate between $2.5 \mathrm{~W}$ and $30 \mathrm{~W}$ is observed. Additionally, a significant increase in thermal conductivity as a result of increasing speed $n$ between $0 \mathrm{~min}^{-1}$ and $3,000 \mathrm{~min}^{-1}\left(a_{c}=327 \cdot \mathrm{g} \mathrm{m} / \mathrm{s}^{2}\right)$ is determined. By simulating the temperature field of a spindle at a load case of $6.000 \mathrm{~min}^{-1}$, a temperature decrease of the rotor from 78.7 ${ }^{\circ} \mathrm{C}$ to $51.1^{\circ} \mathrm{C}$ is achieved (35\%) [Liang 2019b; Liang 2020].

There are comparatively few studies in which eccentrically rotating HP are investigated which are tilted with respect to the axis of rotation. In [Chen 1987], an arrangement of eccentrically rotating and tilted HP with a length of $1,500 \mathrm{~mm}$ and an inner diameter of $19 \mathrm{~mm}$ is investigated up to a speed of $100 \mathrm{~min}^{-1}\left(\mathrm{a}_{\mathrm{c}}=5.7 \cdot \mathrm{g} \mathrm{m} / \mathrm{s}^{2}\right)$. The applied inclination angle is not mentioned in this study. However, an increase in speed from $40 \mathrm{~min}^{-1}$ to $100 \mathrm{~min}^{-1}$ leads to a degressively increase of the heat transfer rate by a factor of three. In [Yau 2011], wickless HP with a distance to the axis of rotation between $75 \mathrm{~mm}$ and $125 \mathrm{~mm}$ are experimentally analyzed. The speed range is $0 \mathrm{~min}^{-1}$ to $580 \mathrm{~min}^{-1}$. The angle between $\mathrm{HP}$ and rotational axis $\alpha_{H P-S}$ is also varied. The setup allows a separate variation of the angles of evaporator, condenser and adiabatic zone between $0^{\circ}$ and $4^{\circ}$. A fundamental increase in heat conductivity is observed due to increasing speeds and distance between HP axis and rotation axis. Compared to a tilted HP, an arrangement parallel to the axis of rotation favors heat conductivity. Increasing $\alpha_{H P-S}$ leads to a further decrease in heat conductivity. The reason for this is the comparatively low centrifugal acceleration in the work. When the HP is tilted, the gravitational acceleration acts disproportionately against the fluid transport. In [Uhlmann 2017] an arrangement of tilted HP for cooling a milling tool of speeds up to $1,200 \mathrm{~min}^{-1}\left(a_{c}=36 \cdot \mathrm{g} \mathrm{m} / \mathrm{s}^{2}\right)$ is shown. There is no discussion of the effect of the tilt on heat conductivity. In this work, however, the favorable effect of tilting on heat transport is underlined.

Furthermore, prototypical designs of cooling systems on the basis of eccentrically rotating HP are known, for which the heat transfer behavior of the applied HP has not been explicitly investigated. Groll et al. [Groll 1978] present an approach for cooling an induction motor with a maximum power of $1.5 \mathrm{~kW}$ and a maximum speed of $5,000 \mathrm{~min}^{-1}$ $\left(a_{c}=2,500 \cdot \mathrm{g} \mathrm{m} / \mathrm{s}^{2}\right)$ using HP inserted axially into the stator and rotor. The authors examined the HP experimentally and found that the fluid return due to the fluid film flattening is sufficient even without tilting the HP. In [Corman 1974], a concept for cooling an induction motor based on eccentrically rotating HP between 4,000 $\mathrm{min}^{-1}$ and 12,000 $\mathrm{min}^{-1}$ $\left(a_{c}=11,137 \cdot \mathrm{g} \mathrm{m} / \mathrm{s}^{2}\right)$ is investigated. This concept also includes HP in the stator. It is shown that a reduction of the rotor temperature occurs due to increasing speed.

An arrangement of eccentrically rotating HP within a geardriven spindle is suggested in the utility model [Yamada 1985]. A lamellar cooling structure is located between the bearings as a heat sink. A cooling medium streams onto a lamellar structure through openings in the housing. This concept has not undergone a technological evaluation. 
In conclusion, it can be stated that the basic operability of eccentrically rotating heat pipes has been determined up to a centrifugal acceleration of $a_{c}=11.137 \cdot \mathrm{g} \mathrm{m} / \mathrm{s}^{2}$. However, isolated studies of eccentrically rotating heat pipes and of their heat transfer behavior have only been conducted up to a centrifugal acceleration of $a_{c}=327 \cdot \mathrm{g} \mathrm{m} / \mathrm{s}^{2}$. Due to the operation of the spindle to be developed (see Fig. 1), maximum centrifugal accelerations between $a_{c} \approx 15,000 \cdot \mathrm{g}$ and $a_{c} \approx 31.000 \cdot \mathrm{g} \mathrm{m} / \mathrm{s}^{2}$ are expected. Therefore, no experimental results or even valid models for the determination of the thermal conductivity at centrifugal accelerations of $a_{c}>$ $327 \cdot \mathrm{g} \mathrm{m} / \mathrm{s}^{2}$ are known. For the determination of the thermal conductivity at higher centrifugal accelerations, the procedure described in the following section is applied.

\section{DETERMINATION OF THE THERMAL CON- DUCTIVITY}

In the following, the utilized test rig is described, as well as the procedure to determine the thermal conductivities of the considered HP. The thermal conductivity is determined by experiments. The considered HP and their specifications are shown in Fig. 5. These are copper HP with a sintered capillary structure (QY-SHP-D6-150SA, type 1) and nickelplated copper HP with axial groove capillary structure (QGSHP-D6-150GN, type 2). Both pipes have deionized water as working fluid. The reason for considering two different HP types is the unpredictable influence of the capillary structure on heat conductivity, which cannot be clearly identified from the current state of research.

\subsection{Experimental setup}

By using the HP test rig (Fig. 6), correlations between the heat conductivity of the HP, the rotational speed $n$, the heat flow rate introduced into the evaporator zone $\dot{Q}_{T}$ and the tilt Manufacturer specifications

\begin{tabular}{|c|c|}
\hline $\begin{array}{l}\text { Type } 1 \\
\text { Pipe material } \\
\text { Capillary structure } \\
\text { Working fluid } \\
\text { Max. transferable } \\
\text { heat flow rate } Q \\
\text { Length } I_{H P} \\
\text { Outer diameter } d_{H P, \text { out }} \\
\text { Inner diameter } d_{H P, \text { in }}\end{array}$ & $\begin{array}{l}\text { QY-SHP-D6-150SA } \\
\text { Copper } \\
\text { Sintered copper } \\
\text { Deionised water } \\
=40 \mathrm{~W} \\
=150 \pm 0.7 \mathrm{~mm} \\
=6.0 \pm 0.05 \mathrm{~mm} \\
=5.75 \pm 0.15 \mathrm{~mm}\end{array}$ \\
\hline $\begin{array}{l}\text { Type } 2 \\
\text { Pipe material } \\
\text { Capillary structure } \\
\text { Working fluid } \\
\text { Max. transferable } \\
\text { heat flow rate } Q \\
\text { Length } I_{H P} \\
\text { Outer diameter } d_{H P, o u t} \\
\text { Inner diameter } d_{H P \text { in }}\end{array}$ & $\begin{array}{l}\text { QG-SHP-D6-150GN } \\
\text { Copper } \\
\text { Grooves } \\
\text { Deionised water } \\
=45 \mathrm{~W} \\
=150 \pm 0.7 \mathrm{~mm} \\
=6.0 \pm 0.05 \mathrm{~mm} \\
=5.70 \pm 0.15 \mathrm{~mm}\end{array}$ \\
\hline
\end{tabular}

\section{Fig. 5 : Heat pipe specifications}

angle $\alpha_{H P-S}$ can be investigated. The selected distances between HP and rotation axis $r_{H P-S}=39 \mathrm{~mm}$ and the HP diameter $d_{H P}=6 \mathrm{~mm}$ correspond approximately to the dimensions as they are to be implemented in the prototype spindle (see Fig. 1). The length of the examined HP $\left(I_{H P}=150\right)$ $\mathrm{mm}$ corresponds to the expected length in the front area of the spindle. A separate examination of longer HP, as they are used in the rear spindle area, is not done in this paper. It can be assumed that the results obtained by examining the short HP can be transferred to longer HP. Instead of two heat sources along the HP axes in the spindle design (Fig. 1 ), a single heat source is applied at one end of the HP for the purpose of design simplification.

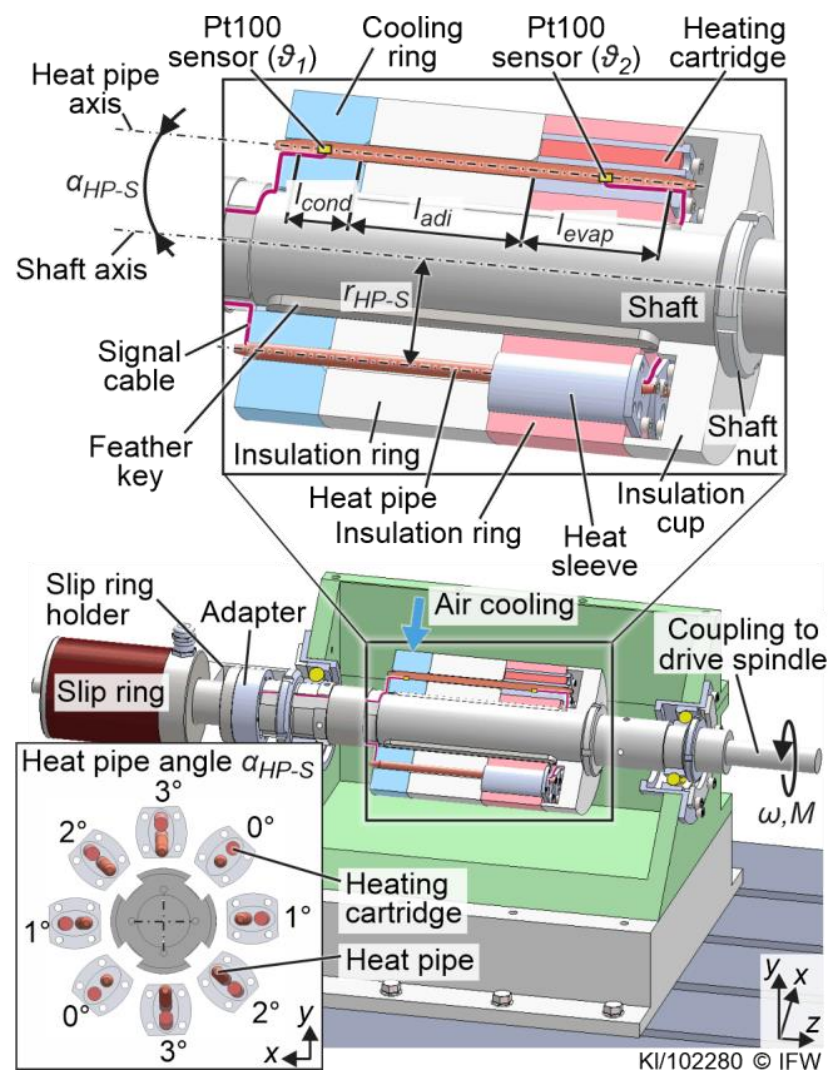

Fig. 6 : Heat pipe test rig

The experimental setup consists of a symmetrical arrangement of eight HP mounted in a system of three cylindrical ring bodies. These rotate around the main shaft axis. Two $\mathrm{HP}$ are each tilted at angles of $\alpha_{H P}=0^{\circ}, 1^{\circ}, 2^{\circ}$ and $3^{\circ}$ to the rotation axis (Fig. 6 , left bottom). The HP themselves are fitted into heat sleeve inserts (Al2024) (fit H7/h6). Heating cartridges (HS-Heizelemente HS/VDE 655/160) with a diameter of $6.5 \mathrm{~mm}$ and a length of $50 \mathrm{~mm}$ are inserted into these inserts (fit H7/h6). Through these cartridges, a definable heat input into the HP is realized. The effective evaporator length levap resulting from the heating sleeve geometry is $52 \mathrm{~mm}$. To increase the heat conductivity between heating cartridges and heat sleeve, thermal paste is applied between these bodies. The heat sleeve inserts are in turn inserted into an insulation ring (diameter $120 \mathrm{~mm}$, width $49.2 \mathrm{~mm}$ ) made of thermally insulating glass hard fabric (Vetronit G11, red body). In order to reduce convection in the area of the heat sleeves, they are protected from air turbulence by means of an insulating cap made of PA6 (outer white body). Temperature sensors (RS PRO Pt100, accuracy class $1 / 3 \mathrm{~B}$ ) are attached to the HP surface using epoxy contact adhesives containing silver (Arctic Silver Thermal Adhesive). Due to the short length of the sensor cables, the sensors are operated with a two-wire connection. One sensor each is located in the middle of the evaporation and condensation zone. The suitability of these positions is confirmed in the literature [Liang 2019a; Ma 2015]. Furthermore, the sensors are mounted orthogonal to the centrifugal force vector. This approximately compensates for the temperature gradient on the HP circumference (see [Mochizuki 1980; Niekawa 1981]).

The insulation ring is mounted one behind the other on the shaft, together with a cooling ring made of Al2024 (blue body) and another insulation ring (PA6, middle white body). All three bodies are connected to the shaft by two feather keys and are axially fixed by a shaft nut. The length of the 
adiabatic zone of the HP is $l_{a d i}=62 \mathrm{~mm}$. The borehole diameters in the insulation ring are one millimeter larger than the HP diameter. Polyurethane foam is inserted into the resulting cavity. This prevents elastic deformation of the pipes due to centrifugal forces during rotation. Moreover, it has also an insulating effect. Unwanted heat transfer in the adiabatic zone is thus reduced. The heat supplied by the heating cartridges is dissipated at the opposite pipe ends via the cooling ring. The HP are connected to this ring via $\mathrm{H} 7 / \mathrm{h} 6$ fittings. The effective width of the condensation zone is Icond $=28.2 \mathrm{~mm}$. Heat is dissipated conductively there through the cooling ring body. To increase the cooling effect, compressed air is blown through a nozzle onto the rotating surface of the cooling ring (air flow rate $350 \mathrm{l} / \mathrm{min}$ ).

The rotary train is mounted in a two-piece housing. The shaft is supported by two ball bearings in a locating bearing/ non-locating bearing arrangement. The bearings are lubricated by bearing grease (Arcanol LOAD 400), which is regularly pressed into the bearings. The test rig shaft is driven by an external spindle (Kessler SN 258 619). The shafts of the drive spindle and the test rig are coupled using a clamping ring hub (Rotex GS-42). The signals of the temperature sensors are transmitted from the rotating system by a slip ring (Rie-Tec SRK 80). The slip ring is coupled to the testrig shaft via a heat-insulating adapter made of PA6. To protect the slip ring housing from rotation, it is screwed to the test rig housing via a slip ring holder. The current required to operate the heating cartridges is also transmitted to the rotating system via the slip ring. Due to the limited number of wires in the slip ring, only simultaneous current and temperature measurement is possible with two HP each. For this reason, two HP displaced by $180^{\circ}$ are equipped with sensors for the experimental procedure. In each case, one type 1 and one type 2 pipe is analyzed simultaneously. Accordingly, only one heating cartridge each is installed. The signals of the temperature sensors which are transmitted via the slip ring are connected to analog input terminals (EL3202) from Beckhoff Automation GmbH \& Co. KG. These are connected to a Beckhoff I-PC via an EtherCAT Coupler (EK1100). The signals are evaluated by the Beckhoff TwinCAT 3 software. The power supply for the two heating cartridges is provided by two separate laboratory isolating transformers (Block BR1000 and EA-STT $2000 \mathrm{~B}$ ). Using two separate power supplies is reasonable, since the heating cartridges have manufacturing-related deviations in their resistances. By separate powering, quantitatively equal heat losses can be generated in both heating cartridges (see next section).

\subsection{Procedure for the determination of the thermal conductivities}

The resulting heat conductivity through a HP is influenced by the heat conductivity of the HP wall material, the heat transport through the working fluid as well as the evaporation or condensation resistance. For the determination of the HP conductivity in this work, these effects are combined by introducing the effective thermal conductivity $\lambda$ eff. Such a simplification is also made in other studies (e.g. [Niekawa 1981]). The determination of $\lambda_{\text {eff }}$ is done according to Eq. 4.

$\lambda_{\text {eff }}=\dot{Q}_{T} \cdot l_{H P, \text { eff }} \cdot\left[A \cdot\left(\vartheta_{2}-\vartheta_{1}\right)\right]^{-1}$

Here, $I_{H P, \text { eff }}$ is the distance between the center of the evaporator and condensation zones. The cross-sectional area $A$ corresponds to the total cross-section of the HP geometry and is $A=\pi \cdot\left(1 / 2 \cdot d_{H P}\right)^{2}=28.3 \mathrm{~mm}^{2}$ for both HP types. The average temperatures of the evaporation and condensation zones $\left(\vartheta_{2}, \vartheta_{1}\right)$ are determined by Pt100 sensors (see Fig. 6 ). The calibration of the four sensors is carried out relatively. The sensor characteristic curves were determined by cooling tests. Using linear equations, the gradients of the characteristic curves of three sensors were adjusted so that their characteristic curve gradients corresponded to the gradient of the fourth sensor (reference sensor). To account for offset values, these values were determined for each sensor at room temperature while the sensors were installed in the test rig. The offset values of the three sensors were again adjusted until the value of the reference sensor was reached. For this procedure, an absolute error of $\pm 0.1 \mathrm{~K}$ per sensor is accepted. However, as only the temperature difference is relevant for the determination of $\lambda_{\text {eff, }}$, the calibration procedure is a suitable means of increasing the accuracy when determining $\lambda_{\text {eff. }}$

The heat loss of the heating cartridges $\dot{Q}_{T}$ is adjusted via the current IHC of a power supply according to Eq. 5, where $R_{T}$ is the resistance of a heating cartridge at room temperature.

$I_{H C}=\sqrt{\dot{Q}_{T} /\left(R_{T}+R_{a d d}\right)}$

However, if a current $I_{H C}$ is applied, the actual current in the heating cartridge is lower. Additional resistances $\Delta R_{E S C}$ within the current supply chain are the reason for this. Additional electrical resistances result from the internal consumption of the power supply unit, line resistances and contact resistances in the slip ring. These resistances were measured in advance of the tests. In addition, the resistances of the heating cartridges increase due to the temperature increases occurring during operation. Temperature-related changes in the heating cartridge resistances $\Delta R_{H C(\vartheta)}$ were experimentally quantified in advance of the measurements at standstill. The heating cartridges were cooled down from $100{ }^{\circ} \mathrm{C}$ to room temperature and their resistances were determined in discrete steps. The maximum deviation between nominal and actual resistance amounts to $2.1 \%$. Preliminary tests have shown that the influence of speed or temperature on the resistance of the slip ring is negligible. The change in resistance of the supply chain $\triangle R_{E S C}$, which is assumed to be constant, as well as the temperature-dependent change of the heating cartridge resistances $\Delta R_{H C(\vartheta)}$, are taken into account for the determination of $I_{H C}$. For this purpose, $\triangle R_{H C(\vartheta)}$ and $\triangle R_{E S C}$ are combined as additional losses Radd according to Eq. 6 and added to the value of $R_{T}$ (see Eq. 5).

$R_{\text {add }}=\Delta R_{H C(\vartheta)}+\Delta R_{E S C}$

In practice, the influence of $\Delta R_{H C(\vartheta)}$ is compensated by a one-time adjustment of the current $I_{\mathrm{HC}}$ once a constant temperature $\vartheta_{2}$ is reached. The reciprocal effect between current and temperature change is hereby not entirely accounted for. However, a large part of the temperature-related change in resistance can be compensated in this way.

The procedure for determining the thermal conductivity is shown in Fig. 7. The determination of the measured variables $\vartheta_{1}$ and $\vartheta_{2}$ is performed at constant speed. During each experiment, the system must be in a thermally stable state. A thermally stable state is characterized by the fact that the values of the temperature $\vartheta_{2}$ remain consistent within $<0.1$ $\mathrm{K}$. After this state is reached, the data acquisition is started and a period of five more minutes passes until the data acquisition is finished. The stationary temperatures $\vartheta_{1}$ and $\vartheta_{2}$ are determined by calculating the arithmetic mean value of the measuring points within the acquisition time span (temperature values steps $\mathrm{i}$ to $\mathrm{j}$ ). 
Temperature value acquisition

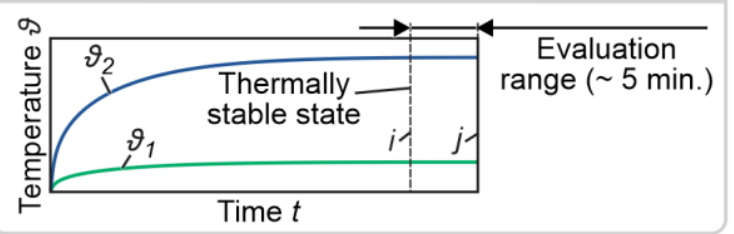

Average temperatures at thermally stable state

Evaporation zone Condensation zone

$$
\vartheta_{2}:=\frac{1}{j} \sum_{i=1}^{j} \vartheta_{2, i} \quad \vartheta_{1}:=\frac{1}{j} \sum_{i=1}^{j} \vartheta_{1, i}
$$

Calculation of thermal conductivity according to Eq. 4

KI/102281ㅇIFW

\section{Fig. 7 : Procedure for thermal conductivity determination}

\section{EVALUATION}

Variation parameters for the experiments are the rotational speed $n$, the introduced heat flow rate $\dot{Q}_{T}$ and the tilt angle between the HP axis and the shaft axis $\alpha_{H P-S}$. For each level of the parameter values $\dot{Q}_{T}$ and $\alpha_{H P-S}$, the speed $n$ is varied in several steps. The heat flow rate $\dot{Q}_{T}$ is varied in two steps $(15 \mathrm{~W}$ and $25 \mathrm{~W})$. These values are based on the expected heat input from the rotor losses into the spindle shaft (see Fig. 1). Since, according to the state of knowledge, a greater effect of centrifugal force on heat conductivity can be expected at lower speeds, the speed increments were reduced in this range. At $4.000 \mathrm{~min}^{-1}$ resonance vibrations occurred. For this reason, no measurements were made at this speed. During the experiments with $\alpha_{H P-S}=1^{\circ}$, resonance vibrations occurred at $6,000 \mathrm{~min}^{-1}$. Instead, a speed of $6,500 \mathrm{~min}^{-1}$ was set. Due to considerable resonance vibrations, no tests could be carried out at $n>8,000 \mathrm{~min}^{-1}$ $\left(a_{c}=2,862 \cdot \mathrm{g} \mathrm{m} / \mathrm{s}^{2}\right)$. Resonance issues are known to occur during the analysis of rotating HP and have also led to a limitation of the achievable speed in other studies (see [Ponnappan 1995; Streby 1996]). During the first repetitive measurements, the slip ring and the fixed bearing were damaged. As a result of this damage and the obtained findings up to that point, additional repetitive measurements were discontinued after consultation with project partners.

Initially, experiments were carried out with $\alpha_{H P-S}=0^{\circ}$. Subsequently, the test rig was modified for the analyses with $\alpha_{H P-S}=1^{\circ}$. Experiments at further steps of $\alpha_{H P-S}\left(2^{\circ}, 3^{\circ}\right)$ were not carried out. The reason for this, apart from the aforementioned vibration issue, is the findings of the experiments leading up to that point. This will be explained in detail in the following discussion of the experimental results.

In Fig. 8, the effective thermal conductivities $\lambda_{\text {eff }}$ of HP type 1 (top) and type 2 (bottom) are shown as a function of speed for $\alpha_{H P-S}=0^{\circ}$. The graphs for $\dot{Q}_{T}=15 \mathrm{~W}$ and $\dot{Q}_{T}=25 \mathrm{~W}$ are shown respectively. Across all graphs, it can be seen that, as in [Niekawa 1981], $\lambda_{\text {eff }}$ decreases regressively with increasing speed. On the one hand, this is due to an increase in the fluid film thickness during the rotation of eccentrically rotating HP. On the other hand, the decrease in wetting of the cylinder or capillary surface facing the axis of rotation leads to the observed decrease of $\lambda_{\text {eff. }}$. At this point, it should be noted that a qualitatively deviating trend of the thermal conductivity as a function of the rotational speed is observed for a comparable setup in [Liang 2020]. In this study, the thermal conductivity increases degressively with increasing speed. The applied maximum speed is $3,000 \mathrm{~min}^{-}$ ${ }^{1}\left(a_{c}=327 \cdot \mathrm{g} \mathrm{m} / \mathrm{s}^{2}\right)$. This degressive decrease is attributed to the increasing fluid pumping effect at higher speeds.
In all cases, the levels of $\lambda_{\text {eff }}$ approach certain values between rotational speeds of $3,000 \mathrm{~min}^{-1}$ and $8,000 \mathrm{~min}^{-1}$. These values were determined by averaging the thermal conductivities of the last four speed steps. In the case of type 1 , this value is $12,188 \mathrm{~W} /(\mathrm{m} \cdot \mathrm{K})$ for a heat flow rate of $\dot{Q}_{T}=15 \mathrm{~W}$. For $\dot{Q}_{T}=25 \mathrm{~W}$ it is $\lambda_{\text {eff }}=12,118 \mathrm{~W} /(\mathrm{m} \cdot \mathrm{K})$. For type 2, the average values of the last four speed steps are $\lambda_{\text {eff }}=7,439 \mathrm{~W} /(\mathrm{m} \cdot \mathrm{K})$ and $\lambda_{\text {eff }}=10,273 \mathrm{~W} /(\mathrm{m} \cdot \mathrm{K})$, respectively. Such an approximation of the thermal conductivity to a certain value was also observed in [Liang 2020]. The reason for this is the flattening of the fluid film that occurs under fast rotation (see Fig. 4). Above a certain speed of rotation, this flattening is entirely developed. The heat transport is then primarily determined by the axial component of the flattening. The higher thermal conductivities of type 1 are again due to the additional surface area resulting from the sinter structure. This correlation has already been stated in other work such as [Niekawa 1981] or [Reay 2014]. The approximation to certain values indicates that the heat transport is limited by the condensation resistance. However, no verification of this is given in the context of this study.

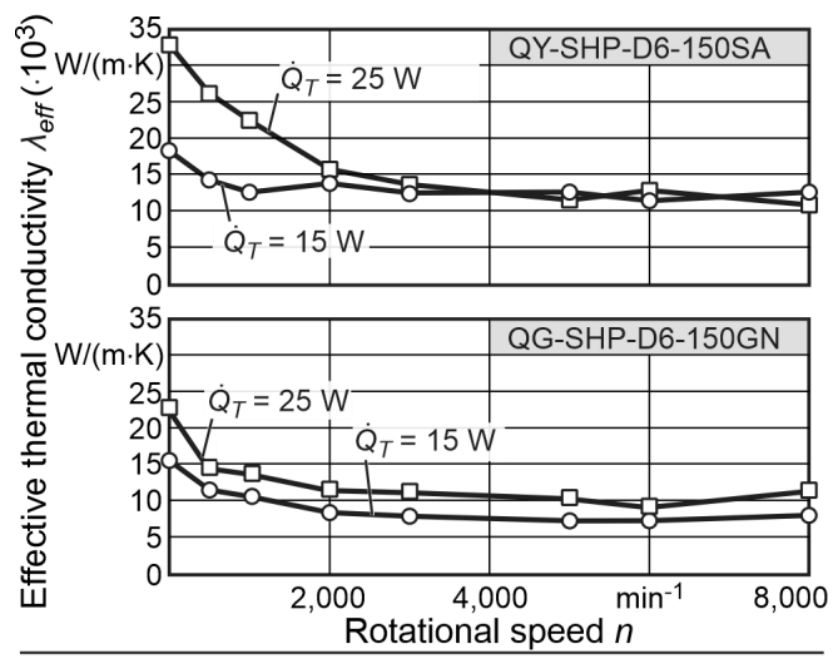

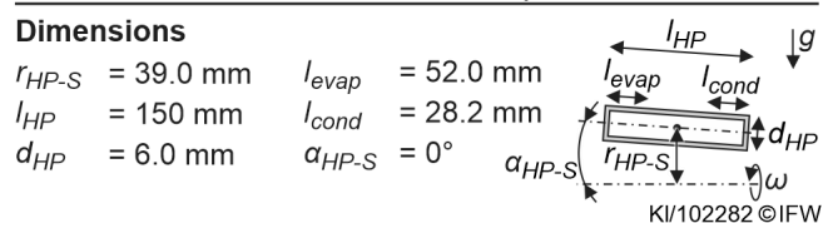

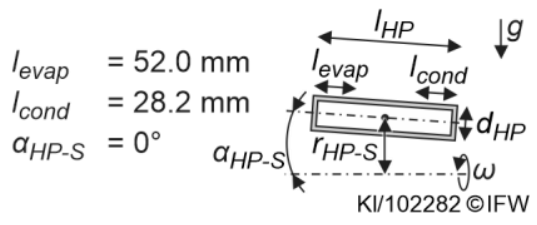

Fig. 8 : Effective thermal conductivities $\lambda_{\text {eff }}$ of heat pipe type 1 (top) and type 2 (bottom) for $\alpha_{H P-S}=0^{\circ}$

At a heat flow rate of $15 \mathrm{~W}$, a value for $\lambda_{\text {eff }}$ of $18,266 \mathrm{~W} /(\mathrm{m} \cdot \mathrm{K})$ for type 1 is determined at standstill. In the case of a heat flow rate of $25 \mathrm{~W}$, the value determined at standstill is significantly higher $(32,774 \mathrm{~W} /(\mathrm{m} \cdot \mathrm{K}))$. For type 2 , the values for heat flow rates of $15 \mathrm{~W}$ and $25 \mathrm{~W}$ at standstill are 15,390 $\mathrm{W} /(\mathrm{m} \cdot \mathrm{K})$ and $22,678 \mathrm{~W} /(\mathrm{m} \cdot \mathrm{K})$ respectively. The observed lower conductivities at low heat flow rates are consistent with the findings of current research (e.g. [Song 2008]). As a result of higher temperatures due to the greater heat input, a faster and more intensive evaporation of the working fluid occurs. This also increases the pressure gradient between the evaporator and condensation zones, which drives the mass transport within the HP. The lower values of type 2 at standstill can be explained by the lower capillary force effect in axial structures in the direction of the tube circumference. As a result, less working fluid is distributed along the circumference of the tube. A higher fluid film thickness as well as drying-out of the upper tube area is the consequence. In addition, type 2 has a smaller capillary surface available for evaporation and condensation. 
For the case of $\alpha_{H P-S}=0^{\circ}$, it is to be expected that the values for $\lambda_{\text {eff }}$ will correspond to the values that the curves approaches from $n=3,000 \mathrm{~min}^{-1}$ even for significantly higher speeds than $n>8,000 \mathrm{~min}^{-1}$. There is currently no evidence in the state of the art to contradict this assumption. Furthermore, it should be noted that the presence of a fine-pored capillary structure promotes heat conductivity, especially at lower speeds. This relativizes the statement in [Asias 2007] (see Section 2.1), according to which the centrifugal force effect dominates the heat transport. The results also indicate that type 1 is preferable to type 2 for implementation in the motor spindle due to the higher conductivities, especially at high speeds.

In Fig. 9, the evaluation of the experiments at a tilt angle of $\alpha H P-S=1^{\circ}$ is shown. The determined courses differ qualitatively clearly from the results of the experiments with $\alpha_{H P-S}$ $=0^{\circ}$. With both HP types, lower thermal conductivities can be determined in the lower speed range than for $\alpha_{H P-S}=0^{\circ}$. The identified higher conductivity of $\mathrm{HP}$ with $\alpha_{H P-S}=0^{\circ} \mathrm{com}$ pared to tilted HP at low speeds is confirmed by the findings in [Yau 2011]. This can be explained by the effect of gravity. The working fluid accumulates in the lower area of the condensation zone. Until $g \leq \omega^{2}$ r applies, the fluid must be returned against gravity by capillary force.

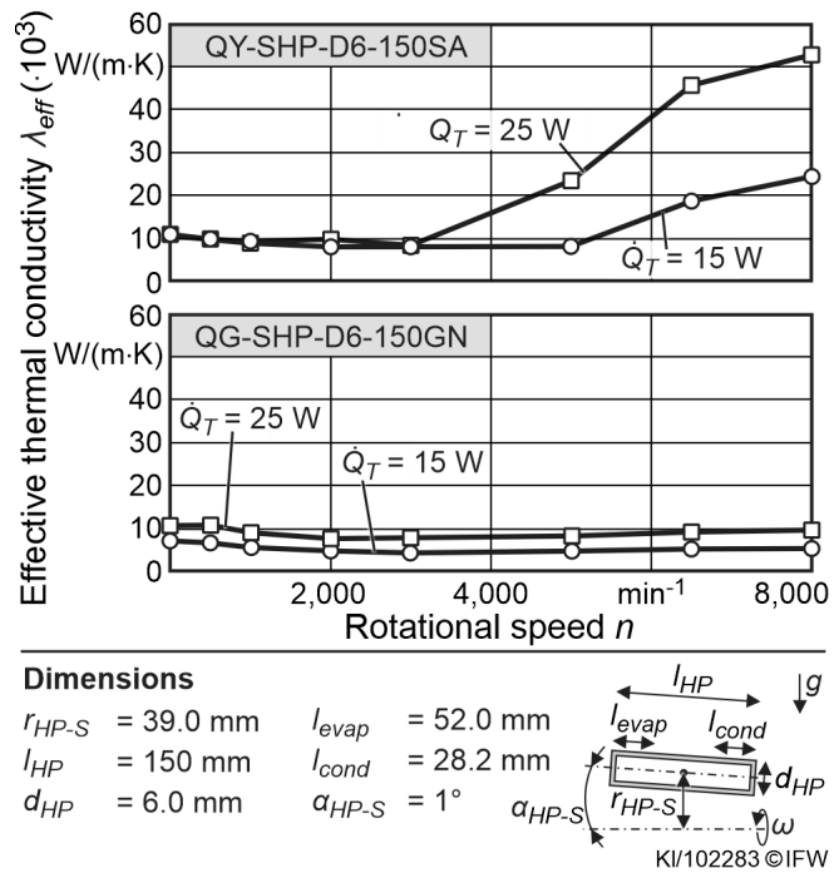

Fig. 9 : Effective thermal conductivities $\lambda_{\text {eff }}$ of heat pipe type 1 (top) and type 2 (bottom) for $\alpha_{H P-S}=1^{\circ}$

The thermal conductivity of type 1 is generally higher than that of type 2 at lower speeds up to $3,000 \mathrm{~min}^{-1}$. Between 0 $\mathrm{min}^{-1}$ and 3,000 $\mathrm{min}^{-1}$, as also observed for $\alpha_{H P-S}=1^{\circ}$, a slight decrease in thermal conductivity $\lambda_{\text {eff }}$ occurs for both types. In this speed range, values between $10,637 \mathrm{~W} /(\mathrm{m} \cdot \mathrm{K})$ and $8,081 \mathrm{~W} /(\mathrm{m} \cdot \mathrm{K})$ can be determined for type 1 at $\dot{Q}_{T}=15$ W. For $\dot{Q}_{T}=25 \mathrm{~W}$, the determined values for $\lambda_{\text {eff }}$ vary between $10,581 \mathrm{~W} /(\mathrm{m} \cdot \mathrm{K})$ and $8,108 \mathrm{~W} /(\mathrm{m} \cdot \mathrm{K})$. For type 1, values between $8,279 \mathrm{~W} /(\mathrm{m} \cdot \mathrm{K})$ and $5,686 \mathrm{~W} /(\mathrm{m} \cdot \mathrm{K})$ can be determined for $\dot{Q}_{T}=15 \mathrm{~W}$. For $\dot{Q}_{T}=25 \mathrm{~W}$, the values determined for $\lambda_{\text {eff }}$ in this speed range are between 10,945 $\mathrm{W} /(\mathrm{m} \cdot \mathrm{K})$ and $8,088 \mathrm{~W} /(\mathrm{m} \cdot \mathrm{K})$.

The significant increase in thermal conductivity of type 1 is noticeable between $3,000 \mathrm{~min}^{-1}$ and 5,000 $\mathrm{min}^{-1}$ ( $\dot{Q}_{T}=25$ W) and between 5,000 $\mathrm{min}^{-1}$ and 6,500 $\mathrm{min}^{-1}\left(\dot{Q}_{T}=15 \mathrm{~W}\right)$, respectively (Fig. 9 top). The higher thermal conductivity at $\dot{Q}_{T}=25 \mathrm{~W}$ can again be attributed to the higher evaporation rate at higher temperatures. An increase in thermal conductivity is also noticeable for type 2 after $3,000 \mathrm{~min}^{-1}$. However, this effect is much smaller than in the case of type 1. The comparatively low thermal conductivities of type 2 indicate that tilting results in a significant accumulation of fluid in the vaporization zone of this type, which in turn acts as thermal resistance. In the case of type 2 such an accumulation is prevented by the significantly larger evaporator surface. The heat is conducted through the sintered structure inside the pipe and can therefore evaporate the fluid more effectively. The large surface of the sintered capillary structure of type 1 thus makes a significant contribution to increasing the evaporation and condensation rate.

Based on the spindle simulation model (see Section 1), it could be shown that thermal conductivities above approximately $8,500 \mathrm{~W} /(\mathrm{m} \cdot \mathrm{K})$ do not lead to a further significant decrease of the shaft temperature. Here, it is shown that, when using HP type 1 at an angle of $\alpha_{H P-S}=0^{\circ}$, a minimal conductivity of about $12,000 \mathrm{~W} /(\mathrm{m} \cdot \mathrm{K})$ can be expected at higher speeds as well. In addition to the dynamic problems described above, this fact is a further reason why no experiments were carried out at larger tilt angles $\alpha_{H P-S .}$ Consequently, type $1 \mathrm{HP}$ was implemented at an angle of $\alpha_{H P-S}=$ $0^{\circ}$ in a preliminary spindle prototype (see [Denkena 2018]).

\section{SUMMARY}

In this paper, the procedure for the determination of thermal conductivities of eccentrically rotating heat pipes was presented. Using a test rig, two different types of heat pipes were investigated. The heat flow rates were varied in two steps (15 W and $25 \mathrm{~W}$ ) and the angle between heat pipe and shaft axis was varied between $0^{\circ}$ and $1^{\circ}$. The tests were initially performed up to a centrifugal acceleration of $2,862 \cdot \mathrm{g} \mathrm{m} / \mathrm{s}^{2}$. It was shown that the heat conductivity is significantly increased by the presence of a sintered structure. The reason for this is the significantly higher surface area available for evaporation and condensation. At high speeds, the values of thermal conductivity approach constant values at an angle of $0^{\circ}$. At an angle of $1^{\circ}$, a sudden and significant increase in conductivity is observed for the heat pipe with sintered structure. It is also shown that the thermal conductivity increases with increasing heat flow rate. Using a spindle simulation model, which is not presented in this paper, it was shown that thermal conductivities above $8,500 \mathrm{~W} /(\mathrm{m} \cdot \mathrm{K})$ do not lead to a further significant reduction of the shaft temperature. This finding also confirms the work of [Groll 1978]. Based on these findings, heat pipes with sintered structure parallel to the axis of rotation were used for an initial spindle prototype. The metrological evaluation of this prototype is presented in [Denkena 2018].

\section{REFERENCES}

[Abele 2011] Abele, E., Korff, D. Avoidance of collisioncaused spindle damages - Challenges, methods and solutions for high dynamic machine tools. CIRP Annals - Manufacturing Technology, 2011, Vol.60, No.1, pp 425-428. DOI 10.1016/j.cirp.2011.03.031

[Asias 2007] Asias, A., et al. Instability of Heat Pipe Performance at large axial accelerations. J. of Heat Transfer, 2007, Vol.129, No.2, pp 137-140. DOI 10.1115/1.2402177

[Chatterjee 2018] Chatterjee, S., et al. Heat transfer in a partially filled rotating pipe with single phase flow. Int. J. of Thermal Sciences, 2018, Vol.125, pp 132-141. DOI 10.1016/j.jijthermalsci.2017.11.024

[Chen 1987] Chen, J., Tu, C. Condenser heat transfer in inclined rotating heat pipe. In: Proc. of the 6th Int. Heat Pipe 
Conf., Grenoble, 25-29 May 1987, Grenoble: CENG-STT, pp 529-534

[Corman 1974] Corman, J., et al. Heat pipe cooled induction motor. IEEE Trans. on Power Apparatus and Systems, 1974, Vol.PAS-93, No.4, pp 1069-1075. DOI 10.1109/TPAS.1974.294051

[Daniels 1975] Daniels, T. C., Al-Jumaily, F. K. Investigations of the factors affecting the performance of a rotating heat pipe. Int. J. of Heat and Mass Transfer, 1975, Vol.18, No.7-8, pp 961-973. DOI 10.1016/0017-9310(75)90190-8

[Denkena 2018] Denkena, B. et al. Cooling potential of heat pipes and heat exchangers within a machine tool spindle. In: Ihlenfeldt S., et al. (eds.) Conf. on Thermal Issues in Machine Tools. Proc.: CIRP sponsored Conf., Dresden, 22-23 March 2018, Auerbach/Vogtland: Verlag Wissenschaftliche Scripten, pp 295-305. ISBN 3-95735-085-9

[Denkena 2020] Denkena, B., et al. Cooling of motor spindles - a review. The Int. J. of Advanced Manufacturing Technology, 2020, Vol.110, No.11-12, pp 3273-3294. DOI 10.1007/s00170-020-06069-0

[Denkena 2020b] Denkena, B. et al. Methodology for thermal optimization of motor spindles. Euspen SIG Meeting on Thermal Issues, Aachen, 26-27 Febr. 2020

[Faghri 1995] Faghri, A. Heat pipe science and technology. Washington: Taylor \& Francis, ISBN 9781560323839

[Gebert 1997] Gebert, K. Ein Beitrag zur thermischen Modellbildung von schnelldrehenden Motorspindeln. Dr.-Ing. Dissertation. Aachen: Shaker, 1997.

[Groll 1978] Groll, M., et al. Heat pipes for cooling of an electric motor. J. of Energy, 1978, Vol.2, No.6, pp 363-367. DOI 10.2514/3.62387

[Hassan 2017] Hassan, H., Harmand, S. An experimental work on the effect of the radius of rotation on the performance of revolving heat pipe (RVHP). Applied Thermal Engineering, 2017, Vol.123, pp 537-545. DOI 10.1016/j.applthermaleng.2017.05.133

[Jankowski 2007] Jankowski, T. Numerical and Experimental Investigations of a Rotating Heat Pipe. Ph.D. Thesis. New Mexico. University of New Mexico, Office of Energy Efficiency and Renewable Energy, 2007.

[Kimura 2005] Kimura, Y. et al. Steady and transient heat transfer characteristics of flat micro heatpipe. Furukawa Review, 2005, Vol.27, pp 3-8

[Li 1993] Li, H. M., et al. Analytical study of the flow and heat transfer in a rotating heat pipe. Heat Recovery Systems and CHP, 1993, Vol.13, No.2, pp 115-122. DOI 10.1016/0890-4332(93)90030-Y

[Li 2018] Li, F., et al. Experimental investigation of single loop thermosyphons utilized in motorized spindle shaft cooling. Applied Thermal Engineering, 2018, Vol.134, pp 229237. DOI 10.1016/j.applthermaleng.2017.11.141

[Li 2020] Li, F., et al. Experimental investigation into rotating loop thermosyphons for cooling shafts of motorized spindles. Heat and Mass Transfer, 2020, DOI 10.1007/s00231020-02919-5

[Li 2020] Li, S.-F., Liu, Z.-h. Parametric study of rotating heat pipe performance: A review. Renewable and Sustainable Energy Reviews, 2020, Vol.117, p 109482. DOI 10.1016/j.rser.2019.109482

[Lian 2016] Lian, W., et al. Numerical investigation on flow and thermal features of a rotating heat pipe. Applied Thermal Engineering, 2016, Vol.101, pp 92-100. DOI 10.1016/j.applthermaleng.2016.02.110

[Liang 2019] Liang, F., et al. An experimental work on thermal features of the miniature revolving heat pipes. Applied
Thermal Engineering, 2019, Vol.146, pp 295-305. DOI 10.1016/j.applthermaleng.2018.09.091

[Liang 2020] Liang, F., et al. Investigation on a grinding motorized spindle with miniature-revolving-heat-pipes central cooling structure. Int. Communications in Heat and Mass Transfer, 2020, Vol.112, p. 104502. DOI 10.1016/j.icheatmasstransfer.2020.104502

[Liang 2019b] Liang, F., Gao, J. et al. A central cooling structure for motorized spindles: Principle and Application. In: Proc. 18th IEEE ITherm. Las Vegas, 28-31 May 2019.

Piscataway: IEEE, pp 1204-1211

[Ling 2001] Ling, J., et al. Experimental Investigations of Radially Rotating Miniature High-Temperature Heat Pipes. J. of Heat Transfer, 2001, Vol.123, No.1, pp 113-119. DOI $10.1115 / 1.1332777$

[Ma 2015] Ma, H. Oscillating heat pipes. New York: Springer. DOI 10.1007/978-1-4939-2504-9

[Mochizuki 1980] Mochizuki, S., Shiratori, T. Condensation heat transfer within a circular Tube under centrifugal Acceleration Field. J. of Heat Transfer, 1980, Vol.102, No.1, pp 158-162. DOI 10.1115/1.3244229

[Niekawa 1981] Niekawa, J., et al. Performance of revolving heat pipes and application to a rotary heat exchanger. J. of Heat Recovery Systems, 1981, Vol.1, No.4, pp 331-338. DOI 10.1016/0198-7593(81)90044-8

[Ponnappan 1995] Ponnappan R., Leland, J. E. High speed rotating heat pipe for aircraft applications. In: Aerospace Atlantic Conf., Dayton, 23-25 May 1995, Warrendale: SAE Int. DOI https://doi.org/10.4271/951437

[Reay 2014] Reay, D. A. et al. Heat pipes. Theory, design and applications. Amsterdam: Elsevier. ISBN 9780080982663

[Salinas 1991] Salinas, D., Marto, P. J. Analysis of an Internally finned rotating heat pipe. Numerical Heat Transfer, Part A: Applications, 1991, Vol.19, No.3, pp 255-275. DOI 10.1080/10407789108944849

[Semena 1991] Semena, M. G., et al. Distribution of heat transfer agent in the capillary structure of rotating heat pipes with a displaced axis of rotation. J. of Engineering Physics, 1991, Vol.60, No.5, pp 642-645. DOI 10.1007/BF00871497

[Song 2003] Song, F., et al. Fluid flow and heat transfer model for high-speed rotating heat pipes. Int. J. of Heat and Mass Transfer, 2003, Vol.46, No.23, pp 4393-4401. DOI 10.1016/S0017-9310(03)00292-8

[Song 2008] Song, F., et al. Heat transfer in the evaporator section of moderate-speed rotating heat pipes. Int. J. of Heat and Mass Transfer, 2008, Vol.51, No.7-8, pp 15421550. DOI 10.1016/j.ijheatmasstransfer.2007.07.020

[Streby 1996] Streby, M. A., et al. Design and testing of a high speed rotating heat pipe. In: Proc. of the 31st Intersociety Energy Conversion Engineering Conf., Washington (DC), 11-16 Aug. 1996. Piscataway, IEEE. DOI 10.1109/IECEC.1996.553939

[Uhlmann 2017] Uhlmann, E., et al. Substitution of coolant by using a closed Internally cooled milling tool. Procedia CIRP 2017, Vol.61, pp 553-557. DOI 10.1016/j.procir.2016.11.267

[Yamada 1985] Yamada, K. Spindle bearing cooling structure (transl.). Patent No. 62-78245

[Yau 2011] Yau, Y. H., Foo, Y. C. Comparative study on evaporator heat transfer characteristics of revolving heat pipes filled with R134a, R22 and R410A. Int. Communications in Heat and Mass Transfer, 2011, Vol.38, No.2, pp 202-211. DOI 10.1016/j.icheatmasstransfer.2010.12.011 